\title{
How can remote sensing data/techniques help us to understand beach hydromorphological behavior?
}

\author{
Joaquim Pais-Barbosa, CICGE, Faculty of Science, University of Porto, jpbarbosa@fc.up.pt \\ Ana Teodoro, CICGE, Faculty of Science, University of Porto, amteodor@fc.up.pt \\ Fernando Veloso-Gomes, IHRH, Faculty of Engineering, University of Porto, vgomes@fe.up.pt \\ Francisco Taveira-Pinto, IHRH, Faculty of Engineering, University of Porto, fpinto@fe.up.pt \\ Hernâni Gonçalves, Faculty of Science, University of Porto, hernani.goncalves@fc.up.pt
}

\begin{abstract}
Beach morphological classification was mainly established for Australian and American microtidal sandy coasts. Different beach morphologies and classification models were presented by several authors. However, parameters such as $\mathrm{Hb}$ (wave breaking height) and $\tan \beta$ (beach slope) are usually unavailable or simply nonexistent for many coastal areas. Therefore, without this information, the morphologic analysis of remotely sensed data from several years is a good approach to identify and to classify beach morphologies. Remote sensing data is an increasingly important component of natural resource monitoring programs and data collection. The aim of this study is to apply different image processing algorithms to different remotely sensed data (aerial photographs and IKONOS image) in order to identify coastal features/patterns. To achieve that, pixel-based, object-based classification algorithms, a hybrid method (called Principal Components Analysis and Histogram), and a pattern recognition approach (using Neural Networks) were applied to remotely sensed data (aerial photographs and IKONOS-2 image). Regarding the results of this work, showing that pixel-based classification (supervised classification algorithms with overall accuracy $>99 \%$ and Kappa Statistic $>0.98$ ) archived better results in comparison with object-based classification; the addition of a NiR band is useful in the classification procedure; and remote sensing data is very useful to identify coastal forms/patterns, helping on the classification of beach morphology, especially in coastal areas where data records are scarce or inexistent.
\end{abstract}

Keywords: Remote sensing, coastal zone, morphology, features, patterns

\section{Introduction}

A coastline represents a dynamic, fragile, physical and biological environment that is constantly changing either at a time scale of days (e.g. between tides) or of years (e.g. global climate change) in response to natural processes as well as human activities. It has held great attraction for man as it provides a variety of resources.

The interface zone between sea and land, present huge challengers in terms of data collection and monitoring methods essentials to the coastal management guidelines definition. The wave-structures/land interactions are rather complex and thus of difficult characterisation. This is not only due to the existence of a dynamic action of non-linear interactions over different time scales, but also to the great difficulty to obtain long data series.

In general, hydrodynamic and morphological data such as $\mathrm{Hb}$, Tan $\beta$, Ws, d50, are unavailable or simply inexistent. However these parameters are vital to the beach 
morphological stage classification. Besides, the understanding of interaction between water and land is scarce, especially concerning coastal forms/patterns formation, maintenance and destruction.

Remote sensing data is an increasingly important component of natural resource monitoring programs. Its utility can be maximised by understanding the constraints and capabilities of the imagery and change detection techniques, related to the monitoring objectives. Remote sensing image classification is one of the most important application domains techniques, and also a form of showing the property of image objects directly and vividly (Dong et al,. 2007).

Using aerial photographs and satellite images analysis, from several years data, seems to be a good approach to study coastal forms/patterns and beach morphology. This work intends to show the importance of remote sensing data and techniques on the improvement of coastal morphology patterns identification regarding the identification of hydromorphologic patterns of breaking waves, sea bottom forms, currents and their relation with local wave and tide regime. A better understanding of the phenomena occurring in the coasts is also expected, namely coastal erosion, helping on the definition of coastal management guidelines and point suitable measures to prevent/minimise coastal problems.

\section{Coastal morphology}

Several beach models and classification were presented in last decades by a number of authors such as Wright and Short (1984), Sunamura (1988), Lippmann and Holman (1990), (Masselink and Short, 1993) and Short (1999).

Short (2006) reports a full range of beach types that occur around the Australian coast, together with the role of waves, tides and sediment in their formation, translated in two parameters: the dimensionless fall velocity $(\Omega)$ and relative tide range (RTR). This classification, in simple terms, includes 15 types of beach systems, distributed over six wavedominated beach types of Wright and Short (1984), three tide-modified types of Masselink and Short (1993), four tide-dominated types and two types in which rock flats of fringing coral reefs dominate the inter to subtidal zone.

Besides these parameters and expressions, the models are represented by comprehensive illustrations where several coastal features/patterns are represented such as: submerged bars (transverse, crescentric, discontinuous, welded and parallel), trough, beach cusp, high tide cusps, mega cusps, erosion cusps, runnel, ridge, mini and strong rip currents, berm crest and erosion berm. Such coastal features/patterns are formed according to wave and tide conditions, sediment budgets, beach slope, sediment size among others.

Based on this assumption, an alternative methodology to classify beach morphologic stage was developed. A first approach was performed and presented by Pais-Barbosa, 2007, PaisBarbosa et al, 2007 and Pais-Barbosa et al, 2009. The methodology consists on a visual identification onscreen in a GIS environment of eight aerial photographs surveys. The visualisation onscreen in a GIS environment methodology allowed the identification and digitalisation of coastal features/patterns. One disadvantage of this methodology is the time consumption to analyse the photographs datasets and to measure the coastal forms/patterns identified. Thus, it is important to develop a methodology that reduces time analyses and that could easily identify and classify the hydroforms and hydromorphologies. 
In the following section four methods based on remote sensing data and image analysis techniques are presented. The first two use aerial photographs and the second two use IKONOS-2 images.

\section{Methods}

\subsection{Pixel-based classification - Supervised and unsupervised}

In the supervised classification, the analyst "supervises" the selection of spectral classes that represent patterns or cover features that they can recognise (it is a "prior decision"). The quality of the recognition is translated by the signature separability function that examines the quality of training site and class signature, before performing the classification. In this work, five training areas were defined for the aerial photographs: Sea, Rip Currents, Breaking Zone, Beachface and Beach.

Three supervised classification algorithms were tested: parallelepiped (with maximum likelihood as tie breaker), minimum distance and maximum likelihood. The parallelepiped classifier is a very simple algorithm, which uses the threshold of each class signature to determine if a given pixel falls within the class or not, and is typically used when a fast implementation is required. In the minimum distance classifier, the distances between the pixel to be classified and each class centre are compared. The pixel is assigned to the class whose centre is the closest to the pixel. The maximum likelihood classification is the most common supervised classification method used with remote sensing image data and is based on Bayesian probability theory (Richards, 1995).

In the unsupervised classification approach, "clustering" algorithms are employed to select spectral classes inherent to the data, more computer-automated (it is a "posterior decision"). In this work, two unsupervised classification algorithms were tested: K-Means and ISODATA (Iterative Self-Organising Data Analysis Techniques). These algorithms are both iterative procedures. The K-Means algorithm samples a subset of the image during cluster means calculation. The ISODATA algorithm is similar to the K-Means algorithm with the distinct difference that the ISODATA algorithm allows for different number of clusters while the KMeans assumes that the number of clusters is known a priori.

\subsection{Object-based classification algorithms}

In the object-oriented approach, the image analysis combines spectral information and spatial information. Therefore, not only the spectral information in the image will be used as classification information, but also the texture and context information in the image will be combined into classification as well (Flanders et al., 2003). In a first stage, the image will be segmented (Table 1) into objects that form the classification units and will be treated as a whole in the classification process.

Table 1 - Segmentation parameters used.

\begin{tabular}{|c|c|c|c|c|c|c|}
\hline Data & Level & Scale & Color & Shape & Smoothness & Compactness \\
\hline 1996 & 2 & 175 & 0.9 & 0.1 & 0.5 & 0.5 \\
\hline 2001 & 3 & 175 & 0.9 & 0.1 & 0.5 & 0.5 \\
\hline
\end{tabular}

The second stage is based on the spectral properties organized hierarchically in classes. Each "super-class" has a class and each "class" has a "sub-class" and thus inherits from one 
or more "super-class" or to its "sub-class". The segments were analysed and classified according to different parameters in the related classes. As a result, the segments with similar characteristics were grouped and classified in the corresponding class. Then, the classification procedure was completed by assigning to the classes color to segments.

\subsection{Principal Components Analysis and Histogram (PCAH) method}

Another alternative method for identifying hydrodynamic forms/patterns is PCAH. The main concept relies on principal components analysis (PCA), which allows for combining the information of the $n$ available spectral bands from the image into an equal number $n$ of principal components (Gonzalez \& Woods, 2002). In this way, each component is obtained from a linear combination of the $n$ spectral bands, and consequently contains information of all spectral bands.

A pre-processing stage is performed prior to the principal component analysis, which allows for an enhancement of the image. The pre-processing is comprised by histogram equalisation, followed by Wiener filtering (Lim, 1990) using a $3 \times 3$ window. The filtering step allows not only to reduce the spiky aspect of the histogram induced by the histogram equalisation, as well as to perform a slight smoothing of the different hydrodynamic forms/patterns existing on the image.

Following the pre-processing stage and the principal components computation, a meaningful segmentation of each principal component can be independently performed, through histogram-based segmentation. The segmentation may be performed either in a manually or automatically manner. The manual procedure is based on a visual identification of classes on the histogram, complemented by some visual inspection on the principal components values. The automatic identification of classes on an histogram may be performed. However The automatic approach is still under development.

\subsection{Pattern recognition approach}

The increasing popularity of neural network models to solve pattern recognition problems has been primarily due to their seemingly low dependence on domain-specific knowledge (relative to model-based and rule-based approaches) and due to the availability of efficient learning algorithms for practitioners to use (Jain et al., 2000).

The use of Artificial Neural Networks (ANNs) to classify remotely sensed data has often resulted in a higher or equal mapping accuracy than the one achieved with traditional classification methodologies or mixture modelling (Benediktsson et al., 1990; Atkinson et al., 1997; Teodoro et al., 2007).

An ANN is a parallel-distributed processor that resembles the human brain by acquiring knowledge through a learning process, and then stores the knowledge in the connection strength between computational units called neurons. An ANN comprises several layers: an input layer, an output layer and one or more hidden layers between them. The hidden layer(s) allow(s) the network to form its own internal representation of the data. If too few nodes are selected, the ANN may not contain sufficient degrees of freedom to form a representation (Haykin, 1999). The network performance depends on the choice of initial weights. Weights connecting the nodes between each layer are initially randomly assigned and adjusted during the learning process to minimize the global error. 
The number of nodes in the input layer is determined by the number of input bands, which in this case consisted of four input nodes - the four IKONOS bands: blue, green, red and NiR. The number of output nodes depends on the number of classes in the classification scheme. In this study, the output layer consisted of five nodes, one for each class: Sea, SuspendedSediments, Breaking-Zone, Beachface and Beach. The dataset was composed by 13775 pixels unequally comprising the five classes, and randomly divided into training $(70 \%$ of each class) and validation subsets (30\% of each class).

Several rules and recommendations exist concerning the calculation of the optimal number of hidden nodes. In this study, the number of hidden nodes was calculated as the number of pairwise separating hyperplanes over the number of classes, according to Dunne and Campbell (1994). The weights of the ANN were estimated, using a statistical software $(R)$, based on the Back-Propagation Algorithm (Haykin, 1999). The stopping criterion was defined to be the maximum number of allowed iterations, which was defined as 300 .

\section{5}

Accuracy assessment

Accuracy assessment is an important step in the classification process. The goal is to quantitatively determine how effectively pixels were grouped into the correct feature classes in the area under investigation. A confusion matrix is a square array of numbers set out in rows and columns which expresses the number of sample units assigned to a particular category relative to the actual category as verified by ground truth information or reference data set. The overall accuracy is given by the division of the total correct by the total number of units (Smits et al., 1999). Kappa analysis is a currently popular multi-variate technique for accuracy assessment (Congalton and Mead, 1983). The Kappa statistic gives a measure that indicates if the confusion matrix is significantly different from a random result. The Kappa value ranges between 0 and 1 . A value of 0 suggests that a given classification is no better than a random assignment of pixels. A value between 0.80 and 1.00 indicates a very good agreement.

\section{Results}

\subsection{Pixel-based classification - Supervised and Unsupervised}

The supervised and unsupervised algorithms referred in the methodology section were applied to two aerial photographs. In the supervised classification all the classes presented a very good separability (>1.9), which is a very good indicator for the classification accuracy.

The performance of the three supervised classification algorithms and the object-oriented classification procedure was evaluated analysing the overall accuracy and the Kappa statistic (Table 2).

Table 2 - Supervised classification accuracy.

\begin{tabular}{|c|c|c|c|c|}
\hline Data & Accuracy Statistics & Parallelepiped & Minimum Distance & Maximum Likelihood \\
\hline \multirow{2}{*}{1996} & Overall Accuracy (\%) & 98.85 & 95.93 & 99.28 \\
\cline { 2 - 5 } & Kappa Statistic & 0.988 & 0.959 & 0.993 \\
\hline \multirow{2}{*}{2001} & Overall Accuracy (\%) & 98.91 & 92.71 & 99.25 \\
\cline { 2 - 5 } & Kappa Statistic & 0.983 & 0.886 & 0.988 \\
\hline
\end{tabular}

The supervised classification algorithm presents a very good performance. The best results 
were found for the maximum likelihood classifier, with an overall accuracy and Kappa statistics of $(99.28 \%, 0.993)$ and $(99.25 \%, 0.988)$ for the 1996 and 2001 aerial photographs, respectively. The results for parallelepiped classifier were similar. However, the minimum distance classifier presented a lower performance. The unsupervised classification algorithms allowed for the identification of different classes such as rip currents, breaking zone, beachface and beach. An example of these results is given in Figure 1.

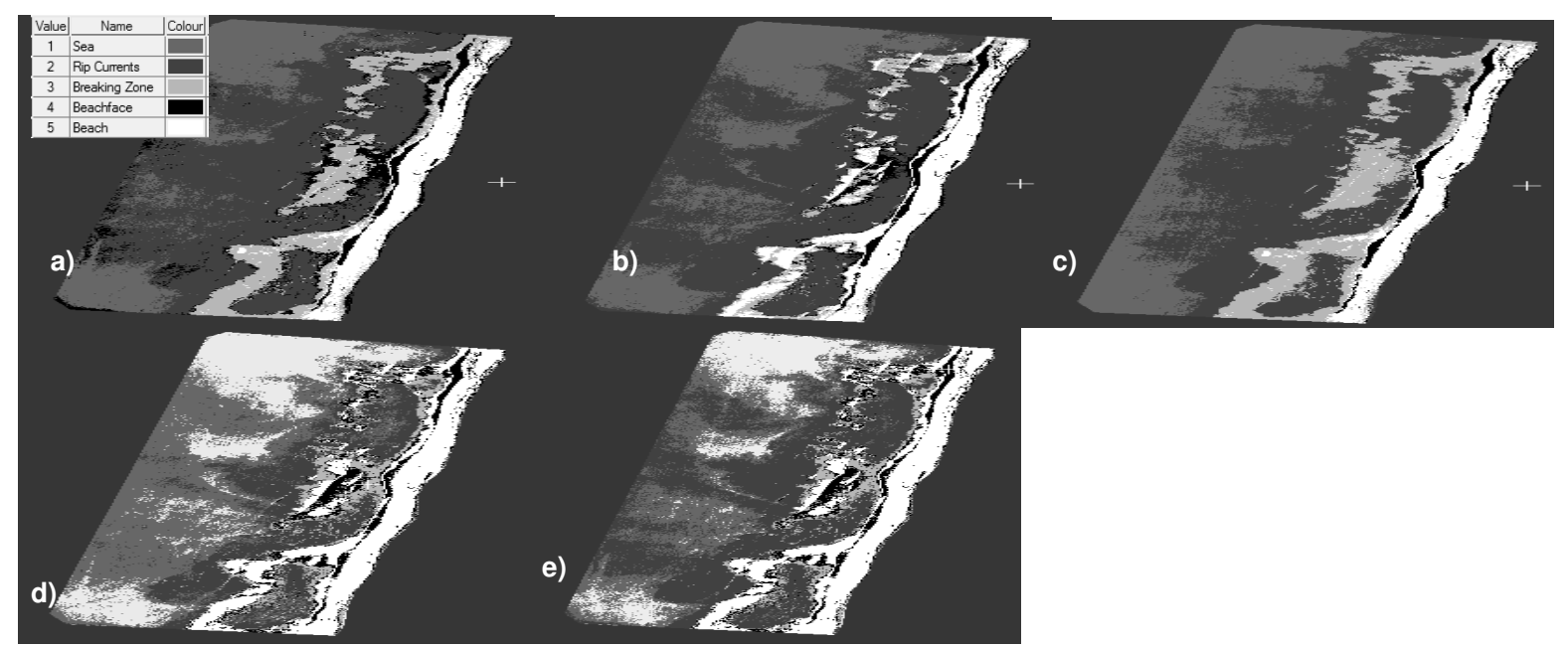

Figure 1 - Results of the supervised classification: a) parallelepiped (with maximum likelihood as tie breaker); b) minimum distance; c) maximum likelihood; and unsupervised classification; d) K-Means; and e) ISODATA algorithms classifiers respectively, applied to the aerial photograph of 2001.

\section{2}

\section{Object-based classification algorithms}

A good performance of the object-oriented classification for the aerial photographs has been achieved. The better result was achieved for the 1996 aerial photograph, with an overall accuracy of $79.75 \%$ and Kappa statistic value of 0.728 . The results achieved for the 2001 aerial photograph presented a lower performance $(69.74 \%$ and 0.603$)$. This method allowed for the identification of several interest classes, as sea, rip currents, breaking zone, beachface and beach (Figure 2 a).

\subsection{Principal Components Analysis and Histogram (PCAH) method}

The PCAH method has been applied to the IKONOS-2 image. For the IKONOS-2 image a fourth band is also available (NiR). The PCAH method allows for an analysis considering the four bands together. The four principal components of the IKONOS-2 image were preprocessed. The proportion of variance explained by principal components 1, 2, 3 and 4 are respectively the following: $94.5 \%, 3.9 \%, 1.3 \%$ and $0.3 \%$. The visual aspect of the principal components is slightly different from what would be expected, due to the pre-processing stage which is performed in the PCAH method. It was observed that each principal component allows for different identifications of the considered classes. The residual variation of the last two principal components still allows for the identification of some (finer) classes.

From the analysis of the segmentation results, it was observed that meaningful results are obtained, in particular regarding the manual identification of classes on the histogram. Using the training classes defined for the pixel-based supervised classification, the proportion of 
correctly classified pixels considering the second principal component were $98 \%, 92 \%, 43 \%$ and $99 \%$, for the classes "sea", "sediments+breaking zone", "beach face" and "beach.

\section{4}

\section{Pattern recognition approach}

In Figure 2 b), the beach features/patterns map obtained through the application of the ANN developed for the IKONOS-2 image is given, with the discrimination of each class identified. The presence of sediment circular forms can be clearly observed. Moreover, it is also possible to discern the maximum swash/runup limit, for the image acquisition moment, defined by the boundary between beach face (wet beach) and beach (dry beach).

The network performance was assessed by estimating the accuracy with which the validation data were classified. The ANN presented a very good performance, demonstrated by the results of the overall accuracy $(98.6 \%)$.

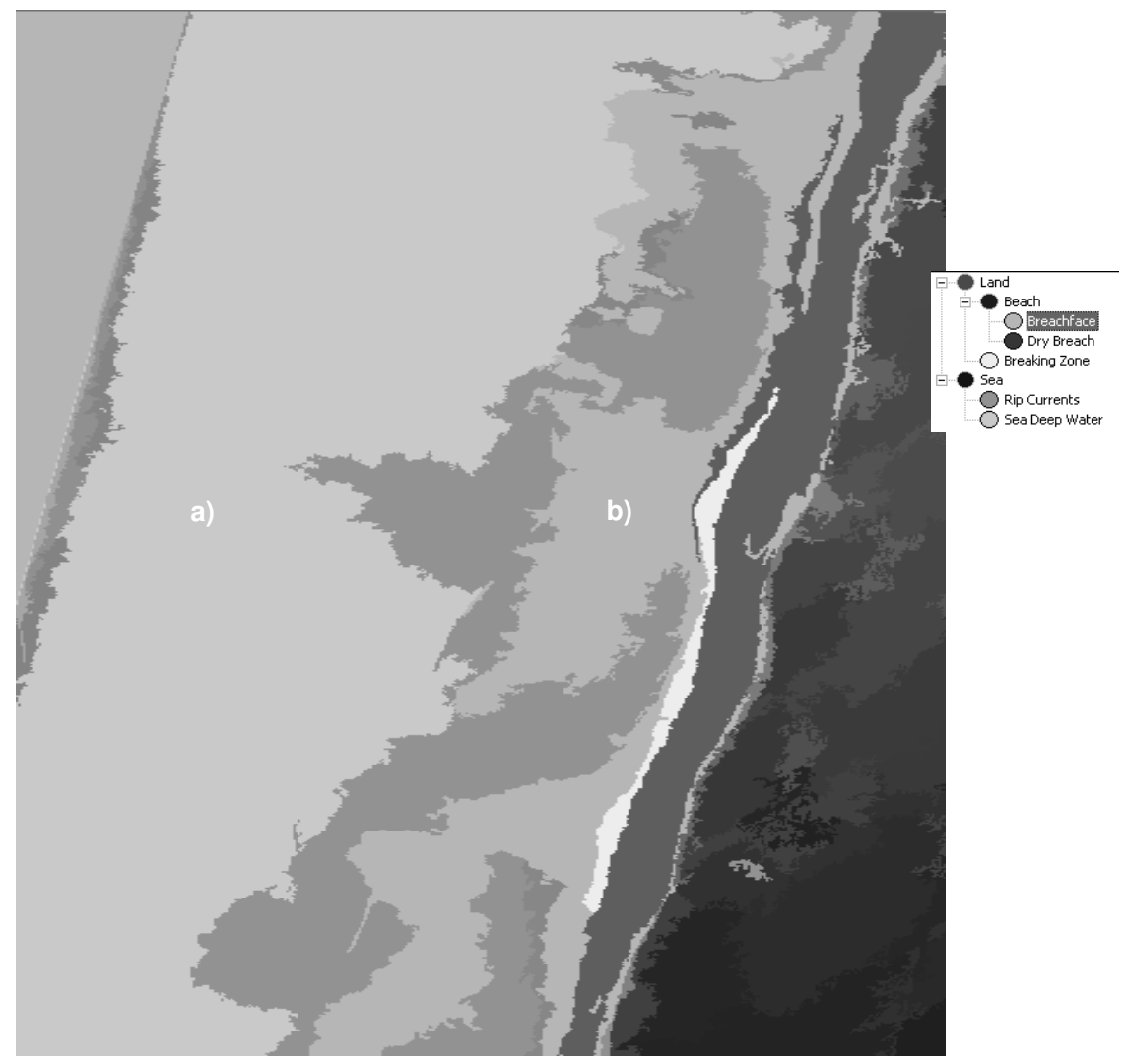

Figure2 - a) Results of the object-based classification for the aerial photograph of 2001; b) Beach features/patterns map for the IKONOS image, obtained through the implemented ANN.

\section{Discussion and conclusions}

Some image classification techniques are presented in this paper. Several conclusions can be drawn regarding the importance of remote sensing data and techniques to understand the beach hydromorphological behavior: (1) the performance achieved were very good allowing the validation of the visual interpretation already performed (Pais-Barbosa, 2007); (2) a pixelbased classification (supervised classification algorithms) is a better approach than an object-based classification; (3) the addition of a NiR band is useful in the classification procedure; (4) the spatial resolution is an important data requisite, demonstrated by the 
results obtained with the pan-sharpened true color image; (5) the PCAH method is a quite interesting and promising method regarding the identification of beach hydromorphological patterns/forms through remotely sensed data; and (5) remote sensing data is very useful to identify coastal forms and patterns, helping on the classification of beach morphology, especially in coastal areas where data records are scarce or inexistent.

This analysis leads to a better understanding of the coastal zone morphodynamics for several wave and tide conditions, helping on the definition of coastal management guidelines and point suitable measures to prevent/minimize coastal problems.

In conclusion, this research demonstrated that the association of remotely sensed data and image processing techniques could be very useful for the identification of beach patterns/forms, as well as helping on beach morphological classification.

In the future, improvements on the classification methodology are expected to be achieved. A software package (stand-alone application) is already under development with the aim of automatically classifying all the datasets.

\section{References}

Atkinson, P.M., Cutler, M.E.J. and Lewis, H.G., 1997. Mapping sub-pixel proportional land cover with AVHRR imagery. International Journal of Remote Sensing, 18, pp. 917-935.

Benediktsson, J.A., Swain, P.H. and Ersoy, O.K., 1990. Neural network approaches versus statistical methods in classification of multisource remote sensing data. IEEE Transactions on Geosciences and Remote Sensing, 28, pp. 540-551.

Congalton, R. G., and Mead, R. A., 1983. A quantitative method to test for consistency and correctness in photointerpretation. Photogrammetric Engineering and Remote Sensing, 49, pp. 69-74.

Dong, G., Zhang Y. and Fan Y., 2007, Remote Sensing Image Classification Algorithm Based on Rough Set Theory. B.-Y. Cao (Ed.): Fuzzy Information and Engineering (ICFIE), ASC 40, pp. 846-851.

Dunne, R.A. and Campbell, N.A., 1994. Some practical aspects of pruning multi-layer perceptron models applied to remotely sensed data. Technical Report 94/06, Murdoch University.

Flanders, D., H. Mryka and P. Joan, 2003. Preliminary evaluation of eCognition object based software for cut block delineation and feature extraction. Canadian Journal of Remote Sensing, 20, pp. 441-452.

Gonzalez, R. C. \& Woods, R.E. (2002). Digital Image Processing. Prentice Hall, 675-683

Haykin, S., 1999. Neural Networks: a comprehensive foundation, 2nd edition. Prentice Hall, pp. 117-173.

Jain, A. K., Duin, R. P. W. and Mao. J., 2000. Statistical Pattern Recognition: A Review. IEEE Transactions on Pattern Analysis and Machine Intelligence, 22 (1), pp. 4-37.

Lippmann, T. C. e Holman, R. A., 1990. The spatial and temporal variability of sand bar morphology. Journal Geophysic Research, 95 (C1), 11575-11590.

Masselink, G., Short, A. D., (1993). The effect of tide range on beach morphodynamics and morphology: A conceptual beach model. Journal of Coastal Research, Vol. 9, pp. 785-800. 
Pais-Barbosa, J., 2007. Hidroformas e Hidromorfologias costeiras locais (in Portuguese). Faculty of Engineering of Porto University (Ed.), PhD Thesis, 2 Vol.

Pais-Barbosa, J., Veloso-Gomes, F., and Taveira-Pinto, F., 2007. Coastal features in the energetic and mesotidal west coast of Portugal. Journal of Coastal Research, SI 50, pp. 459-463.

Pais-Barbosa, J., Veloso-Gomes, F., and Taveira-Pinto, F., 2009. Portuguese Northwest Beach Classification Using Aerial Photographs and GIS Tools. Journal of Coastal Research, SI 56, pp.

Richards, J.A., 1995. Remote Sensing Digital Image Analysis: An Introduction. Springer-Verlag, pp. 265-290.

Short, A. D. (ed.), 1999. Beach and Shoreface Morphodynamics. Chichester, United Kingdom: John Wiley and Sons, 379p.

Short, A. D., 2006, Australian beach systems - nature and distribution. Journal of Coastal Research, 22(1), pp. 11-27.

Smits, P. C., Dellepiane, S. G. and Schowengerdt, R. A., 1999. Quality assessment of image classification algorithms for land-cover mapping: a review and a proposal for a cost-based approach. International Journal of Remote Sensing, 20(8), pp. 1461-1486.

Sunamura, T., 1988. Baech morphologies and their change. In: Horikawa, K. (ed.), Nearshore Dynamics and Coastal Processes. University of Tokoyo Press, pp. 136-166.

Teodoro, A. C., Veloso-Gomes, F., and Gonçalves, H., 2007. Retrieving TSM concentration from multispectral satellite data by multiple regression and artificial neural networks. IEEE Transactions on

Geoscience and Remote Sensing, 45(5), pp. 1342-1350.

Wright, L.D., and Short, A.D., 1984. Morphodynamic variability of surf zones and beaches: a synthesis. Marine Geology 